Louis Emond

Université Laval

\title{
Jeffrey, D. et Harvengt, D. (2017). Ethique et insubordination en éducation. Québec, QC : Presses de I'Université Laval.
}

(Canada)

doi:10.18162/fp.2017.a124

\section{RECENSION}

Pour que l'éthique, comme discipline, soit plus qu'un exercice d'imagination pratiquée dans un cadre universitaire, il faut la confronter à la réalité, aux «faits ». C'est ce que font les auteurs dans Éthique et insubordination en éducation. Pour l'éthique, les faits, ce sont les institutions qui encadrent la pratique enseignante. Dans le cas de l'insubordination, c'est en particulier le droit du travail, qui offre, dans le milieu de l'éducation, une abondante jurisprudence. Parmi un corpus immense, les auteurs ont ainsi choisi, puis analysé, plus d'une vingtaine de cas, vingt-quatre pour être précis, qui se sont retrouvés devant des instances judiciaires ou quasi judiciaires et qui présentent un intérêt particulier afin d'éclairer le lecteur sur les différentes facettes de l'insubordination en enseignement.

\section{Un objet improbable}

Les auteurs classent les décisions dans trois grandes catégories : l'insubordination de type " coups de gueule et entêtement », celle qui touche les « règles et directives contraignantes » et enfin, celle liée à la "pédagogie et aux conflits relationnels». Cet effort de catégorisation des auteurs est des plus éclairants. En effet, il est très rare que des non-juristes prennent comme matière première, pour conduire leur recherche, des documents juridiques. À moins de faire du droit leur objet d'études, les chercheurs en sciences sociales gardent une saine distance par rapport au juridique, souvent perçu comme un monde en lui-même, inaccessible au non-initié.L'ignorance du phénomène juridique par les chercheurs 
en éducation en particulier a d'ailleurs été soulignée par plusieurs auteurs (Carbonnier, 2004; Emond, 2012, 2016; Lemay, 2000). Louvrage de Jeffrey et Harvengt constitue donc une salutaire exception dans le monde des sciences sociales et plus particulièrement des sciences de l'éducation : en regroupant les décisions en catégories qui n'ont pas pour base une logique juridique, bien quelle ne s'y oppose pas, les auteurs mettent en perspective les décisions et les présentent dans un cadre qui favorise leur compréhension pour les pédagogues et les enseignants. L'ouvrage est ainsi un intermédiaire utile entre le droit et la pédagogie, une synthèse qui permet d'illustrer l'ensemble des critères qui sont utilisés par les arbitres et les juges pour évaluer le travail et la conduite d'un enseignant.

\section{Une sélection éclairante}

L'ouvrage s'ouvre sur deux chapitres qui présentent la démarche des auteurs ainsi que les principaux concepts qui serviront tout au long de l'ouvrage. La "perspective démocratique » qu'adoptent les auteurs sert ainsi de référence afin de comparer les décisions avec l'idéal promu par les programmes et, plus généralement, la culture politique québécoise. Ainsi, en introduction, les auteurs défendent clairement "l'idée que la fonction assumée par un membre de la direction d'une école ne lui permet pas de considérer un enseignant comme un subordonné. [...] Dans une école démocratique, le temps est venu de sortir de la relation d'obéissance à un supérieur pour entrer dans celle de respect à l'égard de personnes qui assument des responsabilités différentes des siennes » (p. 13).

Vient ensuite, et cela constitue le véritable cœur du texte, l'analyse des différentes causes qui ont retenu l'attention des auteurs. Pour chacune, il y a un résumé des faits et des arguments du décideur, suivis de l'analyse des auteurs. C'est véritablement de là que vient tout l'intérêt du livre : l'analyse de décisions juridiques à l'aune de principes pédagogiques. C'est en effet dans cet exercice que nous retrouvons les «faits ", que la réflexion éthique peut se confronter à la logique juridique et voir si les arguments éthiques ont un quelconque poids dans la réalité de la relation d'un enseignant avec son employeur. Chaque décision permet d'illustrer différents aspects des relations qu'entretiennent les enseignants avec les élèves, les parents, les directeurs et aussi leurs collègues. Ce n'est d'ailleurs pas sans plaisir que le lecteur passe d'une décision à l'autre. Chacune a des aspects pittoresques ou caricaturaux, que les auteurs ne manquent pas de présenter et qui sont bienvenus pour atténuer l'aridité des concepts juridiques et leur donner, en même temps, plus de consistance. Cela a en plus l'avantage de permettre de plus facilement se souvenir des décisions quand on y fait référence subséquemment dans le texte.

$\mathrm{Au}$ terme de la présentation des décisions, les auteurs synthétisent l'ensemble des remarques et observations qu'ils ont pu faire tout au long de l'ouvrage et dégagent les critères qui définissent l'insubordination ainsi que les normes qui l'encadrent. Ils abordent ainsi des thèmes fort variés, mais qui s'articulent autour de l'éthique de l'enseignant et des normes professionnelles qu'il doit respecter. Cela ramène les auteurs au modèle démocratique qu'ils défendent et à la distance qui la sépare de la réalité du milieu scolaire. Cela leur permet de mettre le doigt sur une problématique sociale beaucoup plus grande, c'est-à-dire celle de l'autorité. Car, au cours du XX $X^{\mathrm{e}}$ siècle, il « y a eu passage d'une autorité fondée sur une anthropologie patriarcale à une autorité fondée sur une anthropologie humaniste qui pose la dignité, c'est-à-dire l'égale valeur de tous les humains, au cœur de toutes relations de responsabilités » (p. 295), ce que les commissions scolaires et les directeurs d'école ne semblent pas tous avoir compris de la même manière. 


\section{Une ouverture sur le droit}

L'ouvrage n'intéressera pas que les enseignants et les pédagogues, il pourrait aussi présenter de l'intérêt pour les juristes. S'ils pouvaient se sentir un peu interdits devant certaines des tournures inhabituelles qu'utilisent les auteurs, certaines explications qui ne semblent pas tout à fait précises, le ton souvent ludique et un système de références étranger, l'intérêt de l'ouvrage demeure. C'est qu'il est une très bonne façon d'observer comment le droit est perçu et compris par des chercheurs d'autres disciplines et par les justiciables eux-mêmes, dans ce cas-ci, les enseignants : par leur analyse, qui s'adresse principalement aux enseignants et aux futurs enseignants, les auteurs permettent d'avoir accès à ce que l'on pourrait nommer la «culture enseignante » lorsquelle est confrontée au droit. Et si un jour on avait l'idée de former minimalement au droit les futurs enseignants, nul doute que l'ouvrage de Jeffrey et Harvengt figurerait en bonne place dans la bibliographie d'un cours sur le sujet.

\section{Références}

Carbonnier, J. (2004). Sociologie juridique. Paris : Presses universitaires de France.

Emond, L. (2012). Droit et pédagogie : définition d'un domaine de recherche. Les Cabiers de droit, 53(3), 523-555. http://dx.doi.org/10.7202/1011938ar

Emond, L. (2016). Exporter le droit : l'exemple de la pédagogie. Dans G. Azzaria (dir.), Les nouveaux chantiers de la doctrine juridique: Actes des 4 e et 5 e Journées d'étude sur la méthodologie et l'épistémologie juridiques. Cowansville, QC : Éditions Yvon Blais.

Lemay, V. (2000). Évaluation scolaire et justice sociale. Montréal, QC : ERPI.

\section{Pour citer cet article}

Emond, L. (2017). Jeffrey, D. et Harvengt, D. (2017). Éthique et insubordination en éducation. Québec, QC : Presses de l'Université Laval. Formation et profession, 25(1), 103-105. http://dx.doi.org/10.18162/fp.2017.a124 